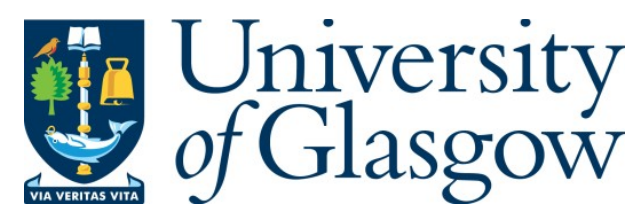

Auckburally, A. (2016) Pulse oximetry and oxygenation assessment in small animal practice. In Practice, 38(2), pp. 50-58.

There may be differences between this version and the published version. You are advised to consult the publisher's version if you wish to cite from it.

http://eprints.gla.ac.uk/129953/

Deposited on: 19 October 2016

Enlighten - Research publications by members of the University of Glasgow http://eprints.gla.ac.uk 


\section{Pulse Oximetry and Assessment of Oxygenation in Small Animal Practice}

\section{Introduction}

Oxygen is essential for cellular respiration of all aerobic organisms. It is therefore important that we can measure the amount of oxygen present within the circulation. In clinical veterinary practice, a non-invasive method of measuring oxygen saturation of arterial blood is necessary for rapid, reliable assessment of a patient's oxygen status, whether anaesthetised or in the intensive care unit. This article will describe the basic technology behind the pulse oximeter, how oxygen is carried within the blood and will also outline some of the pitfalls and limitations of the pulse oximeter.

\section{Technology}

The pulse oximeter was first produced as a prototype in the 1970s, although the technical principles of measuring oxygen in the blood were discovered much earlier. Since then, advances in microprocessor technology and the production of monochromatic light emitting diodes (LEDs) have enabled the development of compact portable pulse oximeters. Pulse oximetry is considered to be essential for the safe conduct of anaesthesia by the Association of Anaesthetists of Great Britain and Ireland and the American Society of Anesthesiologists. These basic standards should be adopted for all anaesthetised veterinary patients, as failure to recognise hypoxaemia is a major cause of preventable death.

The attenuation of light passing through a substance depends, not only on its path length through that substance (Lambert's law), but also on the concentration of that substance (Beer's law). This combined Beer-Lambert Law was published in 1851 and forms the basis of how the pulse oximeter works using spectrophotometry. Conventional pulse oximeters emit two wavelengths of light - usually $660 \mathrm{~nm}$ (red) and $940 \mathrm{~nm}$ (near infrared) - to distinguish between oxygenated $(\mathrm{HbO})$ and deoxygenated $(\mathrm{Hb})$ haemoglobin (Figure 1 ). This means that the pulse oximeter assumes that there are no other non-functioning forms of haemoglobin (see dyshaemoglobinaemias later). Two LEDs are incorporated into the patient 
probe and each emits a single wavelength of light. The probe (Figure 2) is attached to a patient extremity (tongue, lip, toe, prepuce, vulva or ear) (Figure 3) and the light emitted passes through the tissue with the remainder measured by a semiconductor detector (transmitted light). Each LED is energised alternately, many times per second, and there is a brief period of time when neither is energised to enable the detector to account for extraneous light. Some probes use reflected light rather than transmitted light, but reflectance probes have never gained popularity in veterinary medicine and will not be discussed further. The unit must detect arterial haemoglobin oxygen saturation and disregard light absorbed by other tissues e.g. bone, connective tissue and venous blood. The pulsatile nature of arterial blood changes the volume of the tissue in which oxygen saturation of haemoglobin is being measured. In effect, this changes the 'path length' during systole when compared with diastole i.e. during systole, path length is increased because the tissue swells with blood, and so absorption of light increases. Using this characteristic the modern pulse oximeter is able to differentiate between the absorption of light from purely arterial blood in comparison to the rest of the tissue - this technique is a form of pulse plethysmography. The pulse oximeter then uses the ratio of red to infrared light absorbed during pulsatile flow to calculate a saturation value by applying the ratio to a 'look-up table' and reports the value as a percentage. Some pulse oximeters merely show the saturation of haemoglobin as a number, usually in conjunction with a pulse rate and an indicator light to demonstrate that the signal quality is acceptable (Figure 4). Other pulse oximeters, usually those which are incorporated into multi-parameter monitors, also display the plethysmograph trace which is depicted as a pulsatile waveform. The waveform should look distinctly arterial which indicates that the signal is good and the saturation reading is reliable (Figure 5). Pulse rates measured by the pulse oximeter should be compared with actual pulse rates, or with heart rates recorded from real time electrocardiographs incorporated into the same multiparameter monitor, to ensure accuracy.

Most modern pulse oximeters have functions such as alarms for low and high pulse rates, low saturation, poor signal and low battery. Some will also display messages such as 'pulse 
search', 'probe not connected' or 'weak signal' and incorporate a pitch change to give an audible warning when saturation is falling. Many commercially available portable pulse oximeters are now suitable for veterinary use, although few have been validated in veterinary patients. Many are extremely cheap to purchase and all veterinary practices which anaesthetise patients or deal with critically ill patients should have immediate access to this type of monitoring equipment. However, some pulse oximeters are not able to read the very high heart rates encountered in some veterinary species (e.g. birds). Care should be taken when placing probes on the lips or tongue of semi-conscious (critically ill or recovering) animals as the probe may be damaged. Probes should be moved regularly in very small patients to reduce the possibility of thermal tissue damage as a result of heating.

\section{Carriage of oxygen}

In the normal animal, oxygen moves down concentration gradients, from the atmosphere (or inspired gas mixture) down the respiratory tract, into the alveolus, moves across into arterial blood, is disseminated into the systemic circulation, into the tissues and finally is used within the mitochondria of the cells. The amount of oxygen freely dissolved within the plasma is relatively small (approximately $2 \%$ ) and contributes little to the total oxygen content within arterial blood (Equation 1). The presence of haemoglobin significantly increases the amount of available oxygen which is delivered to the tissues (98\%). Haemoglobin is present in large amounts within normal erythrocytes. Each molecule of haemoglobin can bind four oxygen molecules with the binding of each oxygen molecule accelerating the binding of the next (cooperative binding). This results in the distinctive sigmoid shape of the oxygen haemoglobin dissociation curve (Figure 6). The amount of oxygen able to combine with haemoglobin is approximately $1.34 \mathrm{ml}$ of oxygen per gram of haemoglobin.

The abbreviation $\mathrm{PaO}_{2}$ is used to denote the partial pressure of oxygen dissolved in arterial blood and is measured by blood gas analysis. The term $\mathrm{SO}_{2}$ is used to denote the saturation of haemoglobin with oxygen. $\mathrm{A}$ small $\mathrm{p}\left(\mathrm{SpO}_{2}\right)$ indicates that the measurement has been made using a pulse oximeter. $\mathrm{SaO}_{2}$ denotes that an arterial sample has been withdrawn and saturation has been measured using a laboratory based co-oximeter. A co-oximeter is used 
for calibration of pulse oximeters (see later) and also measures all species of haemoglobin i.e. carboxyhaemoglobin, methaemoglobin etc.

The relationship between the amount of free oxygen dissolved in plasma $\left(\mathrm{PaO}_{2}\right)$ and the amount of oxygen bound to haemoglobin $\left(\mathrm{SaO}_{2}\right)$ can be drawn graphically (Figure 6). The curve's position is determined by the $P_{50}$ value which is the partial pressure of oxygen at which the haemoglobin is $50 \%$ saturated at a plasma $\mathrm{pH}$ of 7.4 and a temperature of $37^{\circ} \mathrm{C}$. The $P_{50}$ value differs between the species and is affected by hydrogen ion concentration $(\mathrm{pH})$, temperature, partial pressure of carbon dioxide and 2,3 - diphosphosphoglycerate (a product of cellular metabolism). (Table 1).

Table 1 - Factors affecting the oxygen-haemoglobin dissociation curve

\begin{tabular}{|c|c|}
\hline $\begin{array}{l}\text { Left Shift } \\
\end{array}$ & $\begin{array}{l}\text { Right Shift } \\
\end{array}$ \\
\hline Increased $\mathrm{O}_{2}$ affinity - lungs & Decreased $\mathrm{O}_{2}$ affinity - tissues \\
\hline 仓 $\mathrm{pH}$ & $\sqrt{\mathrm{pH}}$ \\
\hline$\sum\left[\mathrm{H}^{+}\right]$ & 仓 $\left[\mathrm{H}^{+}\right]$ \\
\hline$\sqrt{\mathrm{PaCO}}{ }_{2}$ & 仓 $\mathrm{PaCO}_{2}$ \\
\hline$\S$ temperature & 仓 temperature \\
\hline \& 2,3 -diphosphoglycerate & 仓 2,3-diphosphoglycerate \\
\hline
\end{tabular}

Alterations in hydrogen ion concentration change the affinity of haemoglobin for oxygen and the position of the curve is shifted to the right or left. A left shift of the curve due to a fall in hydrogen ion concentration (an increase in $\mathrm{pH}$ ) reduces the $\mathrm{P}_{50}$ and increases the affinity of haemoglobin for oxygen (encourages oxygen loading), which is the situation in the lung. Conversely, an increase in hydrogen ion concentration (or a fall in $\mathrm{pH}$ ), increases the $\mathrm{P}_{50}$ and decreases the affinity of haemoglobin for oxygen (encourages offloading), which is the situation in the tissues. This is known as the Bohr Effect. If we examine the curve from left to right, due to the sigmoid shape of the curve, the binding of oxygen to haemoglobin is rapid on the steep part of the curve as $\mathrm{PaO}_{2}$ increases and oxygen is loaded efficiently. Once the plateau is reached, saturation is near maximal and further oxygenation of haemoglobin is limited. This physiological adaptation means that haemoglobin is almost fully saturated at relatively low partial pressures of oxygen. Patients breathing oxygen enriched gas mixtures, 
or $100 \%$ oxygen as is often administered during anaesthesia, will have very high $\mathrm{PaO}_{2}$ values, upwards of $500 \mathrm{mmHg}$. If we now examine the curve from right to left, the initial flatter part of the curve (plateau) gives a degree of 'safety', meaning that haemoglobin remains relatively well saturated as $\mathrm{PaO}_{2}$ falls towards $60 \mathrm{mmHg}$. However, once the shoulder of the curve is reached, saturation decreases rapidly. If a problem were to occur (e.g. a pulmonary embolus) that impaired oxygenation, a drop in $\mathrm{PaO}_{2}$ from a value of $500 \mathrm{mmHg}$ down to a value of say $100 \mathrm{mmHg}$ would have very little effect on the pulse oximeter reading: it may drop from an initial value of $100 \%$ down to around $98 \%$ - something that would be unlikely to cause concern. Thus, arterial blood gas analysis gives a more accurate picture of adequacy of oxygenation over the range of $\mathrm{PaO}_{2}$ values observed in clinical practice. The pulse oximeter, however, gives us adequate clinical information because, even although oxygenation is impaired in the animal described above (i.e. the $\mathrm{PaO}_{2}$ is lower than it should be), the patient is not in any real danger until $\mathrm{SpO}_{2}$ drops below about 93\% (the shoulder of the oxyhaemoglobin dissociation curve). The comparison between measuring $\mathrm{PaO}_{2}$ and $\mathrm{SpO}_{2}$ is often described as a person approaching a cliff edge: the $\mathrm{PaO}_{2}$ gives you a warning that you are approaching the edge of the cliff when you are still a kilometre off, and will continue warning you as you get closer; the $\mathrm{SpO}_{2}$, on the other hand, only tells you when you are on the edge of the cliff and about to fall off. $\mathrm{A} \mathrm{PaO}_{2}$ value of less than $60 \mathrm{mmHg}$ and $\mathrm{SaO}_{2}$ below $90 \%$, are usually used to define the term 'hypoxaemia'. For this reason blood gas analysis and measurement of $\mathrm{PaO}_{2}$ is often used in anaesthetic and critical care practice, particularly in patients which may be at risk of hypoxaemia e.g. those with pulmonary disease, or animals which may be otherwise respiratory compromised. Blood gas analysis is beyond the scope of the article and will not be discussed further.

Normal $\mathrm{SpO}_{2}$ should be above $95 \%$ in patients with adequate oxygenation capacity and will usually be $98-100 \%$ in patients receiving supplemental oxygen. Our ability to detect cyanosis as an indicator of hypoxaemia is poor and large amounts of deoxygenated haemoglobin must be present before cyanosis is detectable; $11 \%$ of clinicians were unable to detect 
saturations as low as $75 \%$. Saturation values below approximately $93 \%$ should be investigated urgently for the reasons outlined above. The commonest cause of decreased $\mathrm{SpO}_{2}$ is that the pulse oximeter probe has been in the same place for too long and is compressing the tissue bed. Removing and re-positioning the probe will generally sort the problem. If saturation remains low, the next stage would be check for equipment faults (e.g. failure in oxygen supply; nitrous oxide turned on instead of oxygen). If no fault can be found here, arterial blood gas analysis should (ideally) be performed to confirm whether arterial oxygen tension $\left(\mathrm{PaO}_{2}\right)$ is genuinely low. A discussion of the causes and treatment of hypoxaemia is beyond the scope of this article - see further reading.

\section{Pitfalls and Limitations of Pulse Oximetry}

Although the pulse oximeter is a very useful piece of equipment in veterinary practice, the clinician must appreciate that it does not tell the whole story. By referring to Equation 1, it can be seen that a simple $\mathrm{SpO}_{2}$ value does not tell us the oxygen content of the blood; it just tells us the saturation of the haemoglobin present. Additionally, as oxygen delivery to the tissues relies on cardiac output and oxygen content, pulse oximetry does not inform us of the adequacy of tissue oxygenation either (Equation 2). There are many other considerations when using a pulse oximeter as part of patient monitoring and these are discussed separately below.

1. Anaemia - this cannot be detected by pulse oximetry. Provided the haemoglobin present is functional and saturating normally, it is only the amount of haemoglobin which is reduced and therefore the oxygen content will also be reduced (Equation 1). However, the clinician will not be alerted to this by the pulse oximeter.

2. Dyshaemoglobinaemias - most pulse oximeters disregard all non-functioning species of haemoglobins and only calculate a $\mathrm{Hb}$ : $\mathrm{HbO}$ ratio

a. Carboxyhaemoglobinaemia - as haemoglobin has a very high affinity for carbon monoxide, animals exposed to it (e.g. a house fire) may have high circulating levels or carboxyhaemoglobin $(\mathrm{HbCO})$. The standard pulse 
oximeter is unable to differentiate between $\mathrm{HbO}$ and $\mathrm{HbCO}$ and as absorbances of light are similar, the pulse oximeter reading is driven towards $100 \%$. The pulse oximeter should not be used to assess oxygen status in these animals.

b. Methaemoglobinaemia - the pulse oximeter reading is driven towards $85 \%$ because of the absorption characteristics of methaemoglobin (MetHb). Therefore in patients with oxygen saturation less than $85 \%$, the conventional pulse oximeter will give a falsely high reading and vice versa. Methaemoglobinaemia may be present in cats with paracetamol intoxication, or following the use of some local anaesthetics e.g. prilocaine.

3. Perfusion - the pulse oximeter requires adequate perfusion of an extremity in order to read accurately. Low perfusion states e.g. shock or hypothermia, may interfere with the reading or may result in the pulse oximeter being unable to read at all. Note that hypothermia also leads to shivering - see 4 below. Some drugs e.g. alpha $_{2}$ agonists, cause peripheral vasoconstriction making pulse oximetry impossible or difficult to interpret. The pulse oximeter reading from an extremity such as a toe can be used to estimate blood pressure. A cuff is placed above the extremity and inflated until the pulse oximeter signal disappears and slowly deflated until it reappears in a similar way to measurement using a Doppler flow detector.

4. Vibration and patient movement - this is more of a problem in conscious patients (Figure 7). Any sort of movement of the patient may interfere with the signal acquisition of the pulse oximeter. The equipment should alarm in such an event, but may also give spurious readings which cannot be interpreted. Post-operative shivering is a good example and in these cases it is prudent to measure oxygenation. Hypoventilation associated with anaesthesia may persist and oxygen demand is increased due to shivering. Many modern pulse oximeters are now able to interpret shivering as artefact and disregard it. Again, it is highlighted here that care should be 
taken when using pulse oximeters in conscious patients, since the probes may be easily damaged (chewed), and are expensive to replace.

5. Electromagnetic interference - by far the commonest cause of interference is from strip lighting which can be pulsatile in nature. Modern pulse oximeter probes are shielded to prevent this type of interference. Surgical diathermy and infrared heat lamps are also possible sources of interference. If interference from strip lighting is suspected, cover the probe and see if the reading changes.

6. Pulsatile veins - the pulse oximeter is designed to measure and report the oxygen saturation of haemoglobin in arterial blood. Of course, the saturation of venous blood is much lower as oxygen extraction has occurred in the tissues and the venous blood is being transported back to the lung for oxygenation in the pulmonary capillaries. In some circumstances, venous blood may be pulsatile in nature and lead to erroneous results e.g. tricuspid valve incompetence or atrioventricular dissociation $\left(3^{\circ} \mathrm{AV}\right.$ block).

7. The penumbra effect (partial illumination) - this may lead to erroneously low $\mathrm{SpO}_{2}$ readings. It is caused by a difference in path length for each LED such as may be seen when the probe is placed incorrectly i.e. the tissue thickness varies at the site of probe placement and some emitted light may not pass through the tissue and is detected by the photodetector. Pulsatile venules in extremities may also lead to different path lengths.

8. Pigments and dyes - bilirubin does not appear to interfere with pulse oximetry as it does not absorb light emitted by conventional probes and does not introduce any significant error (Figure 8). However, exogenous dyes which may be used e.g. indocyanine green and methylene blue, will severely interfere with readings due to high absorbance of $660 \mathrm{~nm}$ light. Pulse oximetry readings from animals with pigmented skin e.g. Shar Pei or Chow Chow may be erroneous and some units will fail to read altogether. If available, blood gas analysis should be used to verify readings. 
9. Calibration - although there have been many attempts to calibrate pulse oximeters without the need for human volunteers, the majority of pulse oximeters are calibrated in this way. The healthy volunteer is intentionally desaturated to values as low as $70 \%$ by breathing gas mixtures with low oxygen content. Saturation is measured invasively by withdrawal of arterial samples which are then analysed using a cooximeter. As actual harm may occur it is unethical to desaturate these volunteers further; most pulse oximeters are not calibrated below $70 \%$ and lower values are extrapolated which introduces inaccuracy at these saturation values. Consult the manufacturer information to determine the lowest calibrated saturation.

\section{Pulse oximetry and magnetic resonance imaging (MRI)}

Conventional pulse oximeters must not be used on patients in the MRI unit. Induced voltages created by MRI will interfere with the signal and will also lead to patient burns. The unit of the pulse oximeter will also contain ferrous material and be attracted into the magnetic field. To overcome this, there are pulse oximeters available which transmit light from the base unit through a long fibre optic cable (Figure 9). The main body of the pulse oximeter is situated outside the hazardous area (this may be different for different Tesla units) (Figure 10). The fibre optic probe can then pass through the waveguide port (through the Faraday Cage) (Figure 11) and be attached to the patient. These MRI compatible units are more expensive than conventional ones but basic standards of monitoring apply for patients under general anaesthesia for MRI.

\section{What's New?}

Recent large advances in pulse oximetry technology mean that other species of haemoglobins can be identified and displayed to the clinician in real time (Masimo rainbow ${ }^{\circledR}$ Pulse Co-oximetry ${ }^{\mathrm{TM}}$ ) and patient movement and other artefacts can be removed (Masimo Signal Extraction Technology (SET)). Plethysmographic information generated from a pulse oximeter is also being used to guide fluid therapy by assessment of the waveform during 
mechanical ventilation, known as Pleth Variability Index or PVI®. For a complete discussion of this technology, the reader is referred elsewhere.

\section{Summary}

Pulse oximetry is essential for the safe conduct of anaesthesia and for appropriate care of the critically ill patient. Portable units are very cheap to purchase meaning they are very accessible to veterinary practice. However, values of haemoglobin saturation from pulse oximeters may be affected by many things, and both the veterinary surgeon and the veterinary nurse must be familiar with the limitations and pitfalls to ensure correct interpretation of information.

\section{References \& Further Reading}

Moyle JTB (2002) Pulse Oximetry. BMJ, London, UK

Recommendation for standards of monitoring during anaesthesia and recovery $\left(4^{\text {th }}\right.$ ed) 2007. The Association of Anaesthetists of Great Britain and Ireland.

Schnapp LM and Cohen NH (1990) Pulse oximetry. Use and abuses. CHEST 98 (5), 1244 $-1250$

The Masimo Corporation. http://www.masimo.co.uk/ accessed 30/09/14

McGrotty Y \& Brown A (2013) Blood gases, electrolytes and interpretation 1. Blood gases. In Practice 35, $59-65$. 


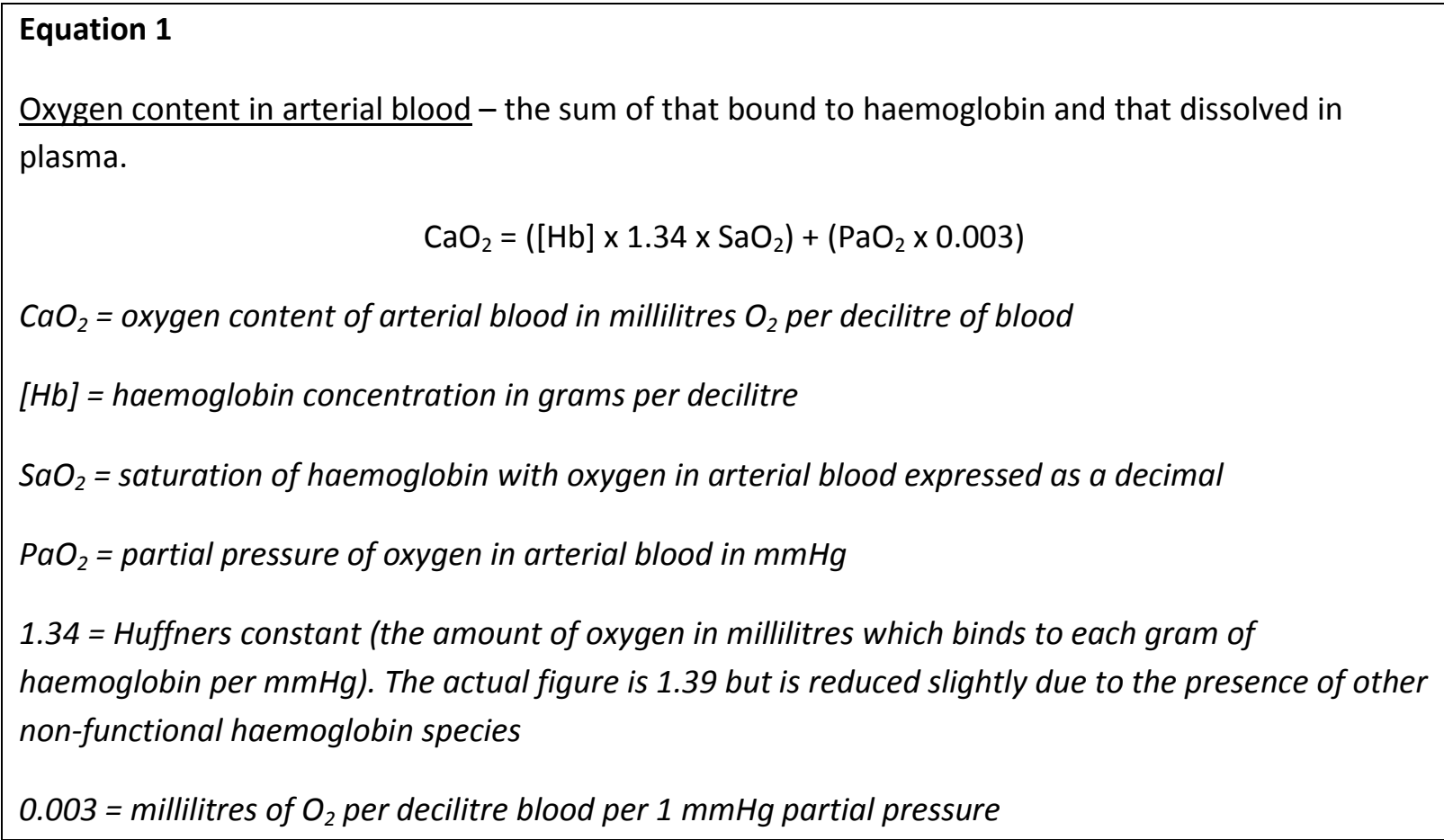

\section{Equation 2}

Oxygen delivery

$$
\mathrm{DO}_{2}=\left(\mathrm{CaO}_{2} \times 10\right) \times \mathrm{CO}
$$

$\mathrm{DO}_{2}=$ oxygen delivery in millilitres per minute

$\mathrm{CaO}_{2}=$ oxygen content of arterial blood in millilitres $\mathrm{O}_{2}$ per decilitre of blood

$\mathrm{CO}=$ cardiac output in litres per minute

Table 1 - Factors affecting the oxygen-haemoglobin dissociation curve

\begin{tabular}{|c|c|}
\hline Left Shift & Right Shift \\
\hline Increased $\mathrm{O}_{2}$ affinity - lungs & Decreased $\mathrm{O}_{2}$ affinity - tissues \\
\hline 仓े $\mathrm{pH}$ & $\sqrt{\mathrm{pH}}$ \\
\hline$\sqrt{n}\left[\mathrm{H}^{+}\right]$ & $\widehat{\vartheta}\left[\mathrm{H}^{+}\right]$ \\
\hline$\sqrt{\Omega} \mathrm{PaCO}_{2}$ & 仓 $\mathrm{PaCO}_{2}$ \\
\hline$\sqrt{ }$ temperature & 仓 temperature \\
\hline I 2,3-diphosphoglycerate & 仓 2,3-diphosphoglycerate \\
\hline
\end{tabular}

